\title{
Dynamique récente des plantations individuelles de palmier à huile au Cameroun
}

\section{Recent dynamics of smallholders's oil palm plantations in Cameroon}

Oléagineux, Corps Gras, Lipides. Volume 7, Numéro 2, 172-4, Mars - Avril 2000, Dossier : Afrique, plantation et développement

\section{Auteur(s) : Robert HIRSCH}

Résumé : Alors que le premier plan palmier camerounais - copie conforme du plan ivoirien - était lancé à la fin des années 60 sous l'impulsion des pouvoirs publics, la plantation individuelle ne constituait pas encore une véritable priorité. Largement dominée par les cinq grandes agro-industries privées (Pamol, Ferme suisse et SAFACAM) ou publiques (CAMDEV et SOCAPALM), la filière huile de palme camerounaise ne s'est réellement ouverte à de nouveaux acteurs2 que vers la fin des années 70 et surtout dans les années 80 . Financées par des crédits publics, ces plantations, qualifiées de villageoises, étaient localisées à la périphérie des agro-industries à qui elles s'engageaient à livrer leurs régimes3 tout en bénéficiant d'un encadrement technique. Au 1er janvier 1999, environ 12400 hectares de plantations relevant de cette catégorie des " plantations villageoises encadrées " étaient encore exploitées ( $33 \%$ dans l'orbite de Pamol, $6 \%$ dans celle de la CAMDEV et $61 \%$ dans les trois secteurs SOCAPALM). Mais ces plantations ayant bénéficié d'une aide publique ne sont guère homogènes. Certains non ruraux - les « élites » selon la terminologie en vigueur au Cameroun - ont réussi à faire financer à bon compte des surfaces qui ne peuvent que difficilement être rangées dans la catégorie des plantations villageoises. Ainsi dans le secteur de Dibombari - le plus important de SOCAPALM - trouve-t-on 4 plantations dont les surfaces déclarées sont supérieures à 100 hectares et 27 autres comprises entre 20 et 100 hectares. Ces plantations de taille moyenne représentent $45 \%$ des superficies réalisées au titre des programmes villageois, qui ont donc été quelque peu dénaturés. Un planteur livrant plus de 1450 tonnes de régimes par an, soit environ 70 millions de F CFA de chiffre d'affaires, peut-il être assimilé au " petit planteur pauvre ", cible désormais privilégiée des bailleurs de fonds ? Mais les liens entre les plantations villageoises stricto sensu et les agro-industries se sont distendus. Des extensions de superficies " hors programme " se sont multipliées et les livraisons aux grandes huileries ont progressivement diminué, les villageois préférant soit assurer par eux-mêmes la transformation de leurs régimes, soit vendre à de petites huileries artisanales leurs produits, parfois mieux rémunérés et surtout payés comptant. Jusqu'au début des années 90 , le prix du kilo de régime fixé par l'administration, sans références clairement perceptibles (cours mondial de I'huile, par exemple), ne satisfaisait personne : ni les grandes sociétés qui le jugeaient trop élevé, ni les producteurs qui le trouvaient trop bas. Lorsque l'insuffisance de la production propre de régimes des sociétés agro-industrielles4 est devenue manifeste, les prix offerts aux plantations villageoises ont été relevés (40 à $50 \mathrm{~F} \mathrm{CFA/kg} \mathrm{contre} 26$ à 31 auparavant) et les livraisons ont partiellement repris et représentent actuellement 12 \% des régimes usinés par les agro-industries. Même si, en 1998, 60 $\%$ des planteurs inscrits de SOCAPALM (1 347 sur 2258 ) ne livraient aucun régime à la société. Pour conclure sur ce secteur villageois encadré (même s'il l'est de moins en moins), on peut considérer que la complémentarité voulue entre ce secteur et les agro-industries n'a pas résisté à l'usure du temps. Les relations entre ces deux ensembles sont désormais fondées davantage sur l'opportunité5, 
voire sur la concurrence, que sur une complémentarité qui, il faut bien le reconnaître, n'a guère été encouragée. Que les planteurs villageois refusent de communiquer leurs surfaces exactes à la société qui les encadre en dit long sur des relations rendues encore plus complexes par l'arrivée de nouveaux venus.

Summary: Smallholders schemes started in the Seventies, with the help of Public Funds. Twenty years later, a new dynamics can be observed in Cameroon. Due to several factors - the economic crisis, falling salaries, a decline in cocoa purchase prices, etc. - this movement has been impulse by new actors, mainly urban, mostly non estate residents and poorly organized. Many of them produce and sell crude palm oil instead of ffb. Beyond this apparent success - all investment requirements are self - financed, without any bank loans - strong competition between smallholders, large oil mills and imported oils can be predicted in a near future. There is an urgent need for a new organization of all the partners involved in the palm oil sector.

Keywords: palm oil, smallholdings, development, organization.

\section{ARTICLE}

\section{Les années 90 : l'arrivée de nouveaux venus}

La crise économique du début des années 90 - et notamment la baisse sensible des salaires - les difficultés du cacao, puis la dévaluation de 1994 ont entraîné non pas un retour à la terre des urbains, mais une certaine prise de conscience que rien ne serait plus comme avant. Cette évolution, à la fois individuelle et collective, concerne des groupes sociaux sans points communs évidents : fonctionnaires et salariés en général, inquiets quant à la réalité et au niveau de leurs retraites futures, commerçants en mal de placements de leurs bénéfices, planteurs de cacao déçus par des prix peu rémunérateurs et une collecte plus ou moins désorganisée, agriculteurs à la recherche de cultures de diversification.

Vis-à-vis de cet ensemble hétérogène, le palmier à huile est apparu comme l'un des remèdes possibles à ces diverses préoccupations par la régularité des revenus qu'il autorise ${ }^{6}$, par la durée du cycle d'exploitation (plus de 20 ans), par les gains escomptés grâce au niveau élevé du prix de l'huile au Cameroun, par une certaine sécurité que peut procurer, aux yeux des non-ruraux, l'activité agricole, etc.

Sur la base des graines vendues, on peut estimer que 15000 à 20000 hectares ont ainsi été plantés de 1994 à 1999 par ces planteurs de $2^{\mathrm{e}}$ génération. Ils ont fait l'objet d'une enquête en 1999, financée par l'AFD, dont l'objet était à la fois de mieux les connaître, d'identifier les conditions dans lesquelles leurs plantations ont été réalisées et d'essayer de sonder leurs aspirations. Réalisée au prix de nombreuses difficultés (planteurs non résidents), cette enquête de Engola Oyep et Bayie Kamanda, du Centre d'études rurales économiques et sociales de l'Université de Yaoundé, a livré de nombreuses informations, mis en évidence des différences régionales significatives et apporté un éclairage intéressant sur ce qui est en passe de devenir un phénomène de société.

Portant sur quatre thèmes (identification du planteur, structure de l'exploitation, production et revenus, perspectives), l'enquête a analysé un échantillon de 108 plantations réparties en trois 
groupes : plantations moyennes ou élites (plus de $10 \mathrm{ha}$ ), plantations satellites des agro-industries (ou encadrées) et plantations isolées dites indépendantes.

Sur les caractéristiques des promoteurs, l'enquête met en évidence :

- leur âge relativement élevé : $66 \%$ ont plus de 45 ans (72\% chez les indépendants) ;

- un absentéisme fort des élites : $67 \%$ ne résident pas à proximité de leur plantation (contre $28 \%$ pour les encadrés et $14 \%$ pour les indépendants) ;

- les allogènes constituent $35 \%$ de l'échantillon ( $80 \%$ dans le Sud-Ouest) ;

- si $27,5 \%$ sont des salariés du secteur public ou privé et $3 \%$ sont commerçants ou occupent une profession libérale, $57 \%$ se déclarent malgré tout agriculteurs ;

- $13 \%$ seulement ont une expérience du travail en palmeraie, ce qui confirme le caractère nouveau de l'activité élaéicole pour la plupart des promoteurs ; en revanche, le niveau d'instruction est très élevé : $61 \%$ des planteurs ont un niveau secondaire ou supérieur.

Sur les exploitations proprement dites, l'enquête permet de mieux cerner cette nouvelle génération de planteurs dont :

- la plantation est inférieure à 5 ha dans $53 \%$ des cas, mais dont 8 ont plus de 50 ha (maximum : 233)

- la terre a été reçue en héritage ( $25 \%$ des cas), achetée ( $28 \%$ ) ou bénéficie d'un droit d'usage (33 $\%)$, le solde résultant d'une combinaison de ces divers modes d'obtention ;

- les plantations sont, dans l'ensemble, très jeunes : $40 \%$ ont été réalisées après 1996 et près de 60 $\%$ depuis 1990, ce qui confirme le caractère récent du phénomène. C'est encore plus net pour les élites dont $57 \%$ des plantations sont postérieures à 1996 (26\% pour les encadrés et $42 \%$ pour les indépendants) ;

- les plants utilisés proviennent à $89 \%$ du secteur moderne (IRAD, sociétés agro-industrielles), ce qui traduit aussi une faible confiance des promoteurs dans les pépiniéristes privés ;

- le coût de création semble être un sujet tabou (seulement $50 \%$ de réponses) si l'on en juge par la diversité des réponses : $20 \%$ des planteurs déclarent un coût nul et l'un d'entre eux annonce 35 millions de F CFA, mais sans que la surface concernée soit précisée ;

- le financement est assuré pour l'essentiel par des fonds propres : $78 \%$ des indépendants et $50 \%$ des élites y ont recours, contre seulement $12 \%$ des encadrés. À signaler également la faiblesse du recours au crédit pour les élites et les indépendants : respectivement 6,7 et 2,8\% des réponses, contre $33 \%$ pour les encadrés (ce qui est logique);

- le recours à la main-d'œuvre salariée, permanente ou non, est généralisé dans les trois groupes identifiés ;

- l'appui technique des services compétents est limité (13,9\% de l'échantillon) et $40 \%$ des planteurs n'ont pas répondu. 
Sur tout ce qui concerne la production et les revenus, sujets sensibles, le taux de réponse est fort variable réduisant par le fait même la portée de l'enquête. Néanmoins, on constate que :

- si $50 \%$ des planteurs vendent uniquement des régimes et $43 \%$ uniquement de l'huile brute, des différences significatives sont perceptibles selon les catégories. Ainsi $47,8 \%$ des élites vendent des régimes, $39 \%$ vendent de l'huile et $9 \%$ combinent ces deux possibilités ${ }^{7}$. En revanche $82 \%$ des encadrés vendent des régimes (c'est leur vocation première) et $78 \%$ des indépendants vendent de I'huile (situés en dehors de l'aide de collecte des agro-industries, ils privilégient alors la meilleure valorisation possible de leurs régimes);

- le palmier est la seule source de revenus pour $26 \%$ de l'effectif enquêté (7 \% des élites, $50 \%$ des encadrés et $14 \%$ des indépendants);

- les motivations pour l'élaéiculture sont conformes aux informations obtenues par ailleurs : $46 \%$ des réponses des élites citent la préparation de la retraite comme raison principale ou annexe, $31 \%$ privilégient l'espoir de gains, le souci d'appropriation foncière n'étant cité que par $3 \%$ des planteurs (différence majeure avec la Côte d'Ivoire) ;

- respectivement 84,3 et 79,6 \% d'entre eux (toutes catégories confondues) entendent accroître leurs superficies et renouveler leurs plantations ;

- une nette majorité ( $83 \%$ ) envisage de procéder à ces investissements totalement ou partiellement sur fonds propres, alors que le crédit, individuel ou collectif, ne recueille que $14,8 \%$ des réponses ;

- la volonté de maîtriser la transformation des régimes est clairement exprimée : 31 \% des planteurs disposent déjà d'une presse (acquise dans $88 \%$ des cas depuis 1994), ce qui tend à montrer que la nouvelle génération n'entend pas rester confinée dans la production primaire (régimes). Si seulement $14 \%$ des encadrés - les moins dynamiques - possèdent une installation, $47 \%$ des élites et $36 \%$ des indépendants ont déjà dépassé ce stade de la production primaire.

Notes

${ }^{1}$ Cet article reflète le point de vue de son auteur et ne peut être considéré comme représentant I'opinion de l'Agence française de développement.

${ }^{2}$ Hormis les nombreux exploitants artisanaux de la palmeraie naturelle.

${ }^{3}$ Et à rembourser les crédits.

${ }^{4}$ Insuffisance due au vieillissement des plantations, à l'accentuation de la saisonnalité de la production et à de nombreuses autres causes parmi lesquelles le vol de régimes.

${ }^{5}$ Je livre mes régimes si le prix me parait intéressant et surtout si je ne trouve pas de meilleure opportunité de les valoriser : telle semble être l'attitude du planteur encadré.

${ }^{6}$ Malgré la saisonnalité forte qui concentre les trois quarts de la production sur 5 à 6 mois.

${ }^{7}$ Pendant la pointe de production, très marquée rappelons-le, aucune presse artisanale ne peut absorber l'intégralité de la production.

${ }^{8}$ D'autant que la forte baisse des cours mondiaux de l'huile depuis le $1^{\mathrm{er}}$ trimestre 1999 (environ 50 
\%) a sensiblement réduit l'efficacité de cette protection.

${ }^{9}$ Cet article constitue la synthèse d'une étude de I'AFD intitulé La filière huile de palme au Cameroun dans une perspective de relance (R. Hirsch, juin 1999, 102 p.) et d'une enquête réalisée sur un échantillon de 108 plantations individuelles par Engola Oyep et Bayie Kamanda du CERES de Yaoundé et financée par l'AFD.

\section{CONCLUSION}

$\mathrm{Au}$ vu des principaux résultats de l'enquête rappelés ci-dessus et des discussions avec les professionnels de la filière huile de palme, il est clair que la dynamique des plantations individuelles est une réalité. Avec un marché intérieur qui est loin d'être saturé, des prix à la consommation élevés, la plantation de palmiers peut se révéler un bon choix. Du moins à court et à moyen terme, car le secteur informel, non taxé et non fiscalisé, profite d'un contexte favorable et notamment de l'affaiblissement passager des sociétés agro-industrielles (en cours de privatisation pour les unes, victimes du vieillissement accéléré de leurs arbres pour les autres). De plus, le marché camerounais demeure très protégé (environ $52 \%$ de droits et taxes sur l'huile brute importée), avantage qui risque de ne pas durer ${ }^{8}$. Or, on ignore tout de la compétitivité du secteur informel qui pourrait, un jour, être confronté à la double concurrence de l'huile importée et des agro-industries.

Néanmoins, sans préjuger de leurs performances agronomiques actuelles et futures, les 15000 à 20 000 hectares réalisés à ce jour par les planteurs individuels n'empêcheront pas la baisse de la production nationale à partir de 2006, rendant improbable la réalisation de l'objectif de production fixé par le gouvernement (250 000 tonnes d'huile en 2010).

L'effort doit donc non seulement être poursuivi mais intensifié, si possible sur des bases différentes.

Jusqu'à présent, comme le notent les auteurs de l'enquête, "l'émergence des palmeraies individuelles relève d'une dynamique individuelle. On note aussi, pour le moment, une très faible structuration du secteur (palmier) ". La création d'une organisation professionnelle de la filière huile de palme figure de manière explicite dans la nouvelle politique agricole camerounaise, mais il semble que ni son contenu ni ses fonctions n'aient fait l'objet de propositions concrètes susceptibles de déclencher une réelle mobilisation des acteurs pressentis. Dans un premier temps, ces derniers gagneraient sans doute à s'organiser par profession afin de se doter d'instances représentatives qui pourraient alors être mandatées pour conduire les négociations en vue de la création d'une véritable organisation interprofessionnelle. En tant que garant de l'intérêt général, l'État pourrait initier ce processus qui prendra du temps étant donné la diversité et la complexité des problèmes à résoudre au préalable. Du temps, il en faudra aussi pour que les nouveaux opérateurs de la filière (repreneurs des sociétés publiques, PMEA, nouveaux planteurs, etc.) définissent leur stratégie et puissent la confronter à celle de leurs concurrents, avant que des positions communes sur les perspectives de I'huile de palme camerounaise à long terme puissent se dégager et faire l'objet d'un véritable consensus.

Le statut d'une future organisation interprofessionnelle devrait, en toute logique, découler des fonctions ou des attributions dont elle aura la responsabilité. Parmi les attributions qui paraissent évidentes, il y a naturellement le suivi statistique des agro-industries et du secteur informel, qui 
devrait être précédé d'un inventaire exhaustif des plantations, des unités de transformation et des multiples intervenants sans lequel aucune gestion raisonnée de la filière palmier n'est envisageable.

La structuration de la filière palmier pose donc toute une série de problèmes dont la diversité et la complexité donnent la mesure, très approximative au demeurant, des efforts que les différents acteurs seront amenés à entreprendre pour asseoir la relance sur des bases saines et durables. $\mathrm{Ce}$ n'est qu'à l'issue de travaux préliminaires validés par de longues négociations entre des partenaires, dont les intérêts, répétons-le une fois encore, sont rarement convergents, que l'on peut espérer voir émerger une véritable organisation professionnelle autonome et capable d'insuffler aux diverses composantes de la filière le dynamisme nécessaire à la réalisation des objectifs globaux assignés au secteur palmier à huile 9 . 